\title{
IMPULSIVE PERIODIC SOLUTIONS FOR SINGULAR PROBLEMS VIA VARIATIONAL METHODS
}

\author{
JUNTAO SUN ${ }^{\bowtie}$ and DONAL O'REGAN
}

(Received 25 September 2011)

\begin{abstract}
In this paper we study impulsive periodic solutions for second-order nonautonomous singular differential equations. Our proof is based on the mountain pass theorem. Some recent results in the literature are extended.
\end{abstract}

2010 Mathematics subject classification: primary 34B15.

Keywords and phrases: Periodic solutions, impulse, singular problems, variational methods, mountain pass theorem.

\section{Introduction}

In this paper we discuss periodic solutions for second-order nonautonomous singular problems

$$
\left\{\begin{array}{l}
u^{\prime \prime}-\frac{b(t)}{u^{\alpha}}=e(t), \quad \text { a.e. } t \in(0, T) \\
u(0)-u(T)=u^{\prime}(0)-u^{\prime}(T)=0
\end{array}\right.
$$

under the impulse conditions

$$
\Delta u^{\prime}\left(t_{j}\right)=I_{j}\left(u\left(t_{j}\right)\right), \quad j=1,2, \ldots, p-1,
$$

where $\alpha>1, \quad b \in \mathbb{C}^{1}([0, T],(0, \infty))$ and $e \in L^{2}([0, T], \mathbb{R})$ are $T$-periodic, $t_{j}, j=$ $1,2, \ldots, p-1$, are the instants when the impulses occur and $0=t_{0}<t_{1}<t_{2}<\cdots<$ $t_{p-1}<t_{p}=T, I_{j}: \mathbb{R} \rightarrow \mathbb{R}(j=1,2, \ldots, p-1)$ are continuous.

Impulse effects occur widely in many evolution processes in which their states are changed abruptly at certain moments in time. For the general aspects of impulsive differential equations, we refer the reader to the classical monograph [18]. Applications of impulsive differential equations with or without delays occur in medicine, population dynamics, chaos theory, and so on; see [8, 16, 17, 21].

(c) 2012 Australian Mathematical Publishing Association Inc. 0004-9727/2012 \$16.00 
Impulsive differential equations have been studied by many authors [4, 11, 19, 20]. Some classical tools have been used to study such problems. These classical techniques include the coincidence degree theory of Mawhin [24], the method of upper and lower solutions [6], some fixed point theorems [7] and variational methods [22, 26, 27]. In 2009, Nieto and O'Regan [22] developed the variational framework for impulsive problems and established existence results for a class of impulsive differential equations with Dirichlet boundary conditions. Sun et al. [26] obtained multiple periodic solutions for second-order perturbed Hamiltonian systems with impulse effects via variational methods.

Singular problems without impulse effects have also been investigated extensively in the literature $[2,3,5,9,10,12-14]$ by using topological methods and variational methods. For example, Boucherif and Daoudi-Merzagui [5] considered a class of singular differential equations and obtained the existence of periodic solutions when the nonlinearity is bounded from above on $u$ by using the mountain pass theorem.

Inspired by [5, 22], in this paper we shall study the existence of periodic solutions for impulsive singular problems. The study of impulsive singular problems is more recent and the number of references is small; see [1, 11, 25]. The tools used in all these references are topological methods. In this paper we prove that problem (1.1)(1.2) has at least one periodic solution by applying variational methods.

Our result is presented as follows.

Theorem 1.1. Assume that:

$\left(\mathrm{S}_{1}\right) \quad b \in \mathbb{C}^{1}([0, T],(0, \infty))$ is $T$-periodic and $b^{\prime}(t) \geq 0$ for all $t \in[0, T]$;

$\left(\mathrm{S}_{2}\right) \quad e \in L^{2}([0, T], \mathbb{R})$ is $T$-periodic and $\int_{0}^{T} e(t) d t<0$;

$\left(\mathrm{S}_{3}\right)$ there exist two constants $m, M$ such that, for any $t \in \mathbb{R}$,

$$
m \leq I_{j}(t) \leq M, \quad j=1,2, \ldots, p-1,
$$

where $m<0$ and $0 \leq M<-1 /(p-1) \int_{0}^{T} e(t) d t$;

$\left(\mathrm{S}_{4}\right)$ for any $t \in \mathbb{R}$,

$$
\int_{0}^{t} I_{j}(s) d s \geq 0, \quad j=1,2, \ldots, p-1 .
$$

Then problem (1.1)-(1.2) has at least one solution.

REMARK 1.2. In fact, it is not difficult to find some functions $I_{j}$ satisfying $\left(\mathrm{S}_{3}\right)$ and $\left(\mathrm{S}_{4}\right)$. For example,

$$
I_{j}(t)=\sin t, \quad t \in \mathbb{R} .
$$

Remark 1.3. Obviously Theorem 1.1 also holds if there is no impulse. In [15], Daoudi-Merzagui studied the existence of periodic solutions for singular differential equations without impulsive effects. In order to apply the method of upper and lower functions, he assumed that the singular nonlinearity $f(t, \cdot)$ is unbounded from above and from below. However, in our paper we consider the case where $f(t, \cdot)$ is only bounded from above (here $f(t, u)=-b(t) / u^{\alpha}$ ). So we extend the result in [15]. Moreover, we also extend the result in [5] to the impulsive case. 


\section{Preliminaries}

Let

$H_{T}^{1}=\left\{u:[0, T] \rightarrow \mathbb{R} \mid u\right.$ is absolutely continuous, $u(0)=u(T)$ and $\left.u^{\prime} \in L^{2}([0, T], \mathbb{R})\right\}$

with the inner product

$$
(u, v)=\int_{0}^{T} u(t) v(t) d t+\int_{0}^{T} u^{\prime}(t) v^{\prime}(t) d t, \quad \forall u, v \in H_{T}^{1} .
$$

The corresponding norm is defined by

$$
\|u\|_{H_{T}^{1}}=\left(\int_{0}^{T}|u(t)|^{2} d t+\int_{0}^{T}\left|u^{\prime}(t)\right|^{2} d t\right)^{1 / 2}, \quad \forall u \in H_{T}^{1} .
$$

Then $H_{T}^{1}$ is a Banach space. (In fact, it is a Hilbert space.)

If $u \in H_{T}^{1}$, then $u$ is absolutely continuous and $u^{\prime} \in L^{2}([0, T], \mathbb{R})$. In this case, $\Delta u^{\prime}(t)=u^{\prime}\left(t^{+}\right)-u^{\prime}\left(t^{-}\right)=0$ is not necessarily valid for every $t \in(0, T)$ and the derivative $u^{\prime}$ may have some discontinuities. This may lead to impulse effects.

Following the ideas of [22], take $v \in H_{T}^{1}$ and multiply the two sides of the equality

$$
-u^{\prime \prime}+\frac{b(t)}{u^{\alpha}}+e(t)=0
$$

by $v$ and integrate from 0 to $T$ :

$$
\int_{0}^{T}\left(-u^{\prime \prime}+\frac{b(t)}{u^{\alpha}}+e(t)\right) v d t=0 .
$$

Note that, since $u^{\prime}(0)-u^{\prime}(T)=0$,

$$
\begin{aligned}
\int_{0}^{T} u^{\prime \prime}(t) v(t) d t & =\sum_{j=0}^{p-1} \int_{t_{j}}^{t_{j+1}} u^{\prime \prime}(t) v(t) d t=\sum_{j=0}^{p-1}\left(u^{\prime}\left(t_{j+1}^{-}\right) v\left(t_{j+1}^{-}\right)-u^{\prime}\left(t_{j}^{+}\right) v\left(t_{j}^{+}\right)\right) \\
& \quad-\sum_{j=0}^{p-1} \int_{t_{j}}^{t_{j+1}} u^{\prime}(t) v^{\prime}(t) d t \\
= & u^{\prime}(T) v(T)-u^{\prime}(0) v(0)-\sum_{j=1}^{p-1} \Delta u^{\prime}\left(t_{j}\right) v\left(t_{j}\right)-\int_{0}^{T} u^{\prime}(t) v^{\prime}(t) d t \\
& =-\sum_{j=1}^{p-1} I_{j}\left(u\left(t_{j}\right)\right) v\left(t_{j}\right)-\int_{0}^{T} u^{\prime}(t) v^{\prime}(t) d t .
\end{aligned}
$$

Combining with (2.1),

$$
\int_{0}^{T} u^{\prime}(t) v^{\prime}(t) d t+\sum_{j=1}^{p-1} I_{j}\left(u\left(t_{j}\right)\right) v\left(t_{j}\right)+\int_{0}^{T} \frac{b(t)}{u^{\alpha}} v(t) d t+\int_{0}^{T} e(t) v(t) d t=0 .
$$

As a result, we introduce the following concept of a weak solution for problem (1.1)-(1.2). 
Definition 2.1. We say that a function $u \in H_{T}^{1}$ is a weak solution of problem (1.1)(1.2) if

$$
\int_{0}^{T} u^{\prime}(t) v^{\prime}(t) d t+\sum_{j=1}^{p-1} I_{j}\left(u\left(t_{j}\right)\right) v\left(t_{j}\right)+\int_{0}^{T} \frac{b(t)}{u^{\alpha}} v(t) d t+\int_{0}^{T} e(t) v(t) d t=0
$$

holds for any $v \in H_{T}^{1}$.

Define the functional $\Phi: H_{T}^{1} \rightarrow \mathbb{R}$ by

$$
\begin{aligned}
\Phi(u):= & \frac{1}{2} \int_{0}^{T}\left|u^{\prime}(t)\right|^{2} d t+\sum_{j=1}^{p-1} \int_{0}^{u\left(t_{j}\right)} I_{j}(s) d s+\int_{0}^{T} b(t)\left(\int_{1}^{u(t)} \frac{1}{s^{\alpha}} d s\right) d t \\
& +\int_{0}^{T} e(t) u(t) d t
\end{aligned}
$$

for every $u \in H_{T}^{1}$. It is easy to verify that $\Phi$ is well defined on $H_{T}^{1}$, continuously differentiable and weakly lower semicontinuous. Moreover, the critical points of $\Phi$ are the weak solutions of problem (1.1)-(1.2).

In the next section, the following version of the mountain pass theorem will be used in our argument.

Theorem 2.2 [23, Theorem 4.10]. Let $X$ be a Banach space and let $\varphi \in C^{1}(X, \mathbb{R})$. Assume that there exist $x_{0}, x_{1} \in X$ and a bounded open neighbourhood $\Omega$ of $x_{0}$ such that $x_{1} \in X \backslash \bar{\Omega}$ and

$$
\max \left\{\varphi\left(x_{0}\right), \varphi\left(x_{1}\right)\right\}<\inf _{x \in \partial \Omega} \varphi(x)
$$

Let

$$
\Gamma=\left\{h \in C([0,1], X): h(0)=x_{0}, h(1)=x_{1}\right\}
$$

and

$$
c=\inf _{h \in \Gamma} \max _{s \in[0,1]} \varphi(h(s)) .
$$

If $\varphi$ satisfies the (PS)-condition (that is, a sequence $\left\{u_{n}\right\}$ in $X$ satisfying $\varphi\left(u_{n}\right)$ is bounded and $\varphi^{\prime}\left(u_{n}\right) \rightarrow 0$ as $n \rightarrow \infty$ has a convergent subsequence), then $c$ is a critical value of $\varphi$ and $c>\max \left\{\varphi\left(x_{0}\right), \varphi\left(x_{1}\right)\right\}$.

\section{Proof of Theorem 1.1}

In order to study problem (1.1)-(1.2), for any $\lambda \in(0,1)$ we consider the modified problem

$$
\left\{\begin{array}{l}
u^{\prime \prime}+b(t) f_{\lambda}(u(t))=e(t), \quad \text { a.e. } t \in(0, T) \\
\Delta u^{\prime}\left(t_{j}\right)=I_{j}\left(u\left(t_{j}\right)\right), \quad j=1,2, \ldots, p-1, \\
u(0)-u(T)=u^{\prime}(0)-u^{\prime}(T)=0
\end{array}\right.
$$


where $f_{\lambda}:[0, T] \times \mathbb{R} \rightarrow \mathbb{R}$ is defined by

$$
f_{\lambda}(u)= \begin{cases}-\frac{1}{u^{\alpha}}, & u \geq \lambda, \\ -\frac{1}{\lambda^{\alpha}}, & u<\lambda .\end{cases}
$$

Let $F_{\lambda}(u)=\int_{1}^{u} f_{\lambda}(s) d s$ and consider the functional

$$
\Phi_{\lambda}: H_{T}^{1} \rightarrow \mathbb{R}
$$

defined by

$$
\begin{aligned}
\Phi_{\lambda}(u):= & \frac{1}{2} \int_{0}^{T}\left|u^{\prime}(t)\right|^{2} d t+\sum_{j=1}^{p-1} \int_{0}^{u\left(t_{j}\right)} I_{j}(s) d s-\int_{0}^{T} b(t) F_{\lambda}(u(t)) d t \\
& +\int_{0}^{T} e(t) u(t) d t .
\end{aligned}
$$

Clearly, $\Phi_{\lambda}$ is well defined on $H_{T}^{1}$, continuously differentiable and weakly lower semicontinuous. Moreover, the critical points of $\Phi_{\lambda}$ are the weak solutions of problem (3.1).

Proof. The proof is divided into four steps.

Step 1. We verify that the functional $\Phi_{\lambda}$ satisfies the Palais-Smale condition.

Let a sequence $\left\{u_{n}\right\}$ in $H_{T}^{1}$ be such that $\Phi_{\lambda}\left(u_{n}\right)$ is bounded and $\Phi_{\lambda}^{\prime}\left(u_{n}\right) \rightarrow 0$. That is, there exist a constant $c_{1}>0$ and a sequence $\left\{\epsilon_{n}\right\}_{n \in \mathbb{N}} \subset \mathbb{R}^{+}$with $\epsilon_{n} \rightarrow 0$ as $n \rightarrow+\infty$ such that, for all $n$,

$$
\left|\int_{0}^{T}\left(\frac{1}{2}\left|u_{n}^{\prime}(t)\right|^{2}-b(t) F_{\lambda}\left(u_{n}(t)\right)+e(t) u_{n}(t)\right) d t+\sum_{j=1}^{p-1} \int_{0}^{u_{n}\left(t_{j}\right)} I_{j}(s) d s\right| \leq c_{1},
$$

and, for every $v \in H_{T}^{1}$,

$$
\left|\int_{0}^{T}\left[u_{n}^{\prime}(t) v^{\prime}(t)-b(t) f_{\lambda}\left(u_{n}(t)\right) v(t)+e(t) v(t)\right] d t+\sum_{j=1}^{p-1} I_{j}\left(u_{n}\left(t_{j}\right)\right) v\left(t_{j}\right)\right| \leq \epsilon_{n}\|v\|_{H_{T}^{1}} .
$$

By a standard argument, it suffices to show that $\left\{u_{n}\right\}$ is bounded when verifying the (PS)-condition.

Taking $v(t) \equiv-1$ in (3.4),

$$
\left|\int_{0}^{T}\left(b(t) f_{\lambda}\left(u_{n}(t)\right)-e(t)\right) d t-\sum_{j=1}^{p-1} I_{j}\left(u_{n}\left(t_{j}\right)\right)\right| \leq \epsilon_{n} \sqrt{T} \quad \text { for all } n .
$$


By $\left(S_{3}\right)$,

$$
\begin{aligned}
\left|\int_{0}^{T} b(t) f_{\lambda}\left(u_{n}(t)\right) d t\right| & \leq \epsilon_{n} \sqrt{T}+\left|\int_{0}^{T} e(t) d t\right|+\sum_{j=1}^{p-1}\left|I_{j}\left(u_{n}\left(t_{j}\right)\right)\right| \\
& \leq \epsilon_{n} \sqrt{T}+\left|\int_{0}^{T} e(t) d t\right|+(p-1) M:=c_{2} .
\end{aligned}
$$

Note that, for any $t \in[0, T], b(t) f_{\lambda}\left(u_{n}(t)\right)<0$. Thus

$$
\int_{0}^{T}\left|b(t) f_{\lambda}\left(u_{n}(t)\right)\right| d t=\left|\int_{0}^{T} b(t) f_{\lambda}\left(u_{n}(t)\right) d t\right| \leq c_{2} .
$$

On the other hand, take, in (3.4),

$$
v(t) \equiv w_{n}(t):=u_{n}(t)-\bar{u}_{n}, \quad \text { where } \bar{u}_{n}=\frac{1}{T} \int_{0}^{T} u_{n}(t) d t .
$$

By [23, Proposition 1.1],

$$
\begin{aligned}
c_{3}\left\|w_{n}\right\|_{H_{T}^{1}} & \geq \int_{0}^{T}\left(w_{n}^{\prime}(t)^{2}-b(t) f_{\lambda}\left(u_{n}(t)\right) w_{n}(t)+e(t) w_{n}(t)\right) d t+\sum_{j=1}^{p-1} I_{j}\left(u_{n}\left(t_{j}\right)\right) w_{n}\left(t_{j}\right) \\
& \geq\left\|w_{n}^{\prime}\right\|_{L^{2}}^{2}-\left(c_{2}+\|e\|_{L^{1}}\right)\left\|w_{n}\right\|_{L^{\infty}}+(p-1) m\left\|w_{n}\right\|_{L^{\infty}} \\
& =\left\|w_{n}^{\prime}\right\|_{L^{2}}^{2}-\left(c_{2}+\|e\|_{L^{1}}-(p-1) m\right)\left\|w_{n}\right\|_{L^{\infty}} \\
& \geq\left\|w_{n}^{\prime}\right\|_{L^{2}}^{2}-c_{4}\left\|w_{n}\right\|_{H_{T}^{1}},
\end{aligned}
$$

where $c_{3}$ and $c_{4}$ are two positive constants. Thus,

$$
\left\|w_{n}^{\prime}\right\|_{L^{2}}^{2} \leq\left(c_{3}+c_{4}\right)\left\|w_{n}\right\|_{H_{T}^{1}} .
$$

Consequently, using the Wirtinger inequality, we see that there exists $c_{5}>0$ such that

$$
\left\|u_{n}^{\prime}\right\|_{L^{2}}^{2} \leq c_{5}
$$

Now suppose that

$$
\left\|u_{n}\right\|_{H_{T}^{1}} \rightarrow+\infty \quad \text { as } n \rightarrow+\infty .
$$

Since (3.5) holds, we have, passing to a subsequence if necessary, that either

$$
\begin{array}{ll}
M_{n}:=\max u_{n} \rightarrow+\infty & \text { as } n \rightarrow+\infty \\
m_{n}:=\min u_{n} \rightarrow-\infty & \text { or } \\
& \text { as } \rightarrow+\infty .
\end{array}
$$

(i) Assume that the first possibility occurs. By $\left(\mathrm{S}_{3}\right)$ and the fact that $f_{\lambda}<0$,

$$
\begin{aligned}
& \int_{0}^{T}\left(b(t) F_{\lambda}\left(u_{n}(t)\right)-e(t) u_{n}(t)\right) d t-\sum_{j=1}^{p-1} \int_{0}^{u_{n}\left(t_{j}\right)} I_{j}(s) d s \\
& \quad \geq \int_{0}^{T}\left(\left(\int_{1}^{u_{n}(t)} b(t) f_{\lambda}(s) d s\right)-e(t) u_{n}(t)\right) d t-(p-1) M M_{n}
\end{aligned}
$$




$$
\begin{aligned}
= & \int_{0}^{T}\left(\left(\int_{1}^{M_{n}} b(t) f_{\lambda}(s) d s-\int_{u_{n}(t)}^{M_{n}} b(t) f_{\lambda}(s) d s\right)-e(t) u_{n}(t)\right) d t-(p-1) M M_{n} \\
= & \int_{0}^{T} b(t) F_{\lambda}\left(M_{n}\right) d t-\int_{0}^{T} M_{n} e(t) d t-\int_{0}^{T}\left(\int_{u_{n}(t)}^{M_{n}}\left(b(t) f_{\lambda}(s)-e(t)\right) d s\right) d t \\
& -(p-1) M M_{n} \\
\geq & F_{\lambda}\left(M_{n}\right) \int_{0}^{T} b(t) d t-M_{n} \int_{0}^{T} e(t) d t+\int_{0}^{T}\left(M_{n}-u_{n}(t)\right) e(t) d t \\
& -(p-1) M M_{n} \\
\geq & F_{\lambda}\left(M_{n}\right) \int_{0}^{T} b(t) d t-M_{n} \int_{0}^{T} e(t) d t-\|e\|_{L^{1}}\left\|M_{n}-u_{n}\right\|_{C}-(p-1) M M_{n} .
\end{aligned}
$$

Thus, using Sobolev and Poincaré's inequalities,

$$
\begin{aligned}
&-\left((p-1) M+\int_{0}^{T} e(t) d t\right) M_{n} \\
& \leq \int_{0}^{T}\left(b(t) F_{\lambda}\left(u_{n}(t)\right)-e(t) u_{n}(t)\right) d t-\sum_{j=1}^{p-1} \int_{0}^{u_{n}\left(t_{j}\right)} I_{j}(s) d s+\sqrt{T}\|e\|_{L^{1}}\left\|u_{n}^{\prime}\right\|_{L^{2}} \\
&-F_{\lambda}\left(M_{n}\right) \int_{0}^{T} b(t) d t \\
&= \int_{0}^{T}\left(b(t) F_{\lambda}\left(u_{n}(t)\right)-e(t) u_{n}(t)\right) d t-\sum_{j=1}^{p-1} \int_{0}^{u_{n}\left(t_{j}\right)} I_{j}(s) d s+\sqrt{T}\|e\|_{L^{1}}\left\|u_{n}^{\prime}\right\|_{L^{2}} \\
&-\frac{\int_{0}^{T} b(t) d t}{\alpha-1}\left(\frac{1}{M_{n}^{\alpha-1}}-1\right) .
\end{aligned}
$$

From (3.3), (3.5) and the fact that $1 / M_{n}^{\alpha-1} \rightarrow 0$ as $n \rightarrow+\infty$, we see that the right-hand side of the above inequality is bounded, which is a contradiction.

(ii) Assume that the second possibility occurs, that is, $m_{n} \rightarrow-\infty$ as $n \rightarrow+\infty$. We replace $M_{n}$ by $m_{n}$ in the preceding arguments, and we also get a contradiction.

Therefore, $\Phi_{\lambda}$ satisfies the Palais-Smale condition.

Step 2. Let

$$
\Omega=\left\{u \in H_{T}^{1} \mid \min _{t \in[0, T]} u(t)>1\right\}
$$

and

$$
\partial \Omega=\left\{u \in H_{T}^{1} \mid u(t) \geq 1 \text { for all } t \in(0, T), \exists t_{u} \in(0, T): u\left(t_{u}\right)=1\right\} .
$$

We show that there exists $d>0$ such that $\inf _{u \in \partial \Omega} \Phi_{\lambda}(u) \geq-d$ whenever $\lambda \in(0,1)$.

For any $u \in \partial \Omega$, there exists some $t_{u} \in(0, T)$ such that $\min _{t \in[0, T]} u(t)=u\left(t_{u}\right)=1$. 
By (3.2), $\left(\mathrm{S}_{4}\right)$ and extending the functions by $T$-periodicity,

$$
\begin{aligned}
\Phi_{\lambda}(u)= & \int_{t_{u}}^{t_{u}+T}\left(\frac{1}{2}\left|u^{\prime}(t)\right|^{2}-b(t) F_{\lambda}(u(t))+e(t) u(t)\right) d t+\sum_{j=1}^{p-1} \int_{0}^{u\left(t_{j}\right)} I_{j}(s) d s \\
\geq & \frac{1}{2} \int_{t_{u}}^{t_{u}+T}\left|u^{\prime}(t)\right|^{2} d t+\frac{1}{\alpha-1} \int_{t_{u}}^{t_{u}+T} b(t)\left(1-\frac{1}{u(t)^{\alpha-1}}\right) d t \\
& \quad+\int_{t_{u}}^{t_{u}+T} e(t)(u(t)-1) d t+\int_{t_{u}}^{t_{u}+T} e(t) d t \\
\geq & \frac{1}{2} \int_{t_{u}}^{t_{u}+T}\left|u^{\prime}(t)\right|^{2} d t+\int_{t_{u}}^{t_{u}+T} e(t)(u(t)-1) d t+\int_{t_{u}}^{t_{u}+T} e(t) d t .
\end{aligned}
$$

By the Schwarz inequality,

$$
\Phi_{\lambda}(u) \geq \frac{1}{2}\left\|(u(\cdot)-1)^{\prime}\right\|_{L^{2}}^{2}-\|e\|_{L^{2}} \cdot\|(u(\cdot)-1)\|_{L^{2}}-\|e\|_{L^{1}} .
$$

Applying Poincaré's inequality to $u(\cdot)-1$,

$$
\Phi_{\lambda}(u) \geq \frac{1}{2}\left\|u^{\prime}\right\|_{L^{2}}^{2}-\gamma\|e\|_{L^{2}} \cdot\left\|u^{\prime}\right\|_{L^{2}}-\|e\|_{L^{1}},
$$

where $\gamma=\gamma\left(t_{u}\right)$. The above inequality shows that

$$
\Phi_{\lambda}(u) \rightarrow+\infty \quad \text { as }\left\|u^{\prime}\right\|_{L^{2}} \rightarrow+\infty .
$$

Since $\min _{t \in[0, T]} u(t)=1$, we have that $\|u(\cdot)-1\|_{H_{T}^{1}} \rightarrow+\infty$ is equivalent to $\left\|u^{\prime}\right\|_{L^{2}} \rightarrow$ $+\infty$. Hence

$$
\Phi_{\lambda}(u) \rightarrow+\infty \quad \text { as }\|u\|_{H_{T}^{1}} \rightarrow+\infty, \forall u \in \partial \Omega,
$$

which shows that $\Phi_{\lambda}$ is coercive. Thus it has a minimising sequence. The weak lower semicontinuity of $\Phi_{\lambda}$ yields

$$
\inf _{u \in \partial \Omega} \Phi_{\lambda}(u)>-\infty .
$$

It follows that there exists $d>0$ such that $\inf _{u \in \partial \Omega} \Phi_{\lambda}(u)>-d$ for all $\lambda \in(0,1)$.

Step 3. We prove that there exists $\lambda_{0} \in(0,1)$ with the property that, for every $\lambda \in\left(0, \lambda_{0}\right)$, any solution $u$ of problem (3.1) satisfying $\Phi_{\lambda}(u)>-d$ is such that $\min _{u \in[0, T]} u(t) \geq \lambda_{0}$, and hence $u$ is a solution of problem (1.1)-(1.2).

Assume, to the contrary, that there are sequences $\left\{\lambda_{n}\right\}_{n \in \mathbb{N}}$ and $\left\{u_{n}\right\}_{n \in \mathbb{N}}$ such that:

(i) $\lambda_{n} \leq 1 / n$;

(ii) $u_{n}$ is a solution of (3.1) with $\lambda=\lambda_{n}$;

(iii) $\Phi_{\lambda_{n}}\left(u_{n}\right) \geq-d$;

(iv) $\min _{t \in[0, T]} u_{n}(t)<1 / n$.

Since $f_{\lambda_{n}}<0$ and $\int_{0}^{T}\left(b(t) f_{\lambda_{n}}\left(u_{n}(t)\right)-e(t)\right) d t=0$,

$$
\left\|b(\cdot) f_{\lambda_{n}}\left(u_{n}(\cdot)\right)\right\|_{L^{1}} \leq c_{6}, \quad \text { for some constant } c_{6}>0 .
$$


Hence

$$
\left\|u_{n}^{\prime}\right\|_{L^{\infty}} \leq c_{7}, \quad \text { for some constant } c_{7}>0 .
$$

From $\Phi_{\lambda_{n}}\left(u_{n}\right) \geq-d$, it follows that there must exist two constants $l_{1}$ and $l_{2}$, with $0<l_{1}<l_{2}$ such that

$$
\max \left\{u_{n}(t) ; t \in[0, T]\right\} \subset\left[l_{1}, l_{2}\right] .
$$

If not, $u_{n}$ would tend uniformly to 0 or $+\infty$. In both cases, by $\left(\mathrm{S}_{2}\right)-\left(\mathrm{S}_{3}\right)$ and (3.6),

$$
\Phi_{\lambda_{n}}\left(u_{n}\right) \rightarrow-\infty \quad \text { as } n \rightarrow+\infty,
$$

which contradicts $\Phi_{\lambda_{n}}\left(u_{n}\right) \geq-d$.

Let $\tau_{n}^{1}, \tau_{n}^{2}$ be such that, for $n$ large enough,

$$
u_{n}\left(\tau_{n}^{1}\right)=\frac{1}{n}<l_{1}=u_{n}\left(\tau_{n}^{2}\right) .
$$

Multiplying the differential equation in (3.1) by $u_{n}^{\prime}$ and integrating the equation on $\left[\tau_{n}^{1}, \tau_{n}^{2}\right]$, or on $\left[\tau_{n}^{2}, \tau_{n}^{1}\right]$,

$$
\begin{aligned}
\Psi & :=\int_{\tau_{n}^{1}}^{\tau_{n}^{2}} u_{n}^{\prime \prime}(t) u_{n}^{\prime}(t) d t+\int_{\tau_{n}^{1}}^{\tau_{n}^{2}} b(t) f_{\lambda_{n}}\left(u_{n}(t)\right) u_{n}^{\prime}(t) d t \\
& =\int_{\tau_{n}^{1}}^{\tau_{n}^{2}} e(t) u_{n}^{\prime}(t) d t .
\end{aligned}
$$

It is easy to verify that

$$
\Psi=\Psi_{1}+\frac{1}{2}\left(u_{n}^{\prime 2}\left(\tau_{n}^{2}\right)-u_{n}^{\prime 2}\left(\tau_{n}^{1}\right)\right)
$$

where

$$
\Psi_{1}=\int_{\tau_{n}^{1}}^{\tau_{n}^{2}} b(t) f_{\lambda_{n}}\left(u_{n}(t)\right) u_{n}^{\prime}(t) d t .
$$

From $\left(S_{2}\right)$, (3.6) and (3.7) it follows that $\Psi$ is bounded, and consequently $\Psi_{1}$ is bounded.

On the other hand, it is easy to see that

$$
b(t) f_{\lambda_{n}}\left(u_{n}(t)\right) u_{n}^{\prime}(t)=\frac{d}{d t}\left(b(t) F_{\lambda_{n}}\left(u_{n}(t)\right)\right)-b^{\prime}(t) F_{\lambda_{n}}\left(u_{n}(t)\right) .
$$

Thus, by $\left(\mathrm{S}_{1}\right)$,

$$
\begin{aligned}
\Psi_{1} & =b\left(\tau_{n}^{2}\right) F_{\lambda_{n}}\left(l_{1}\right)-b\left(\tau_{n}^{1}\right) F_{\lambda_{n}}\left(\frac{1}{n}\right)-\int_{\tau_{n}^{1}}^{\tau_{n}^{2}} b^{\prime}(t) F_{\lambda_{n}}\left(u_{n}(t)\right) d t \\
& \leq b\left(\tau_{n}^{2}\right) F_{\lambda_{n}}\left(l_{1}\right)-b\left(\tau_{n}^{1}\right) F_{\lambda_{n}}\left(\frac{1}{n}\right)-\frac{1}{\alpha-1} \int_{\tau_{n}^{1}}^{\tau_{n}^{2}} b^{\prime}(t)\left(\frac{1}{l_{2}{ }^{\alpha-1}}-1\right) d t .
\end{aligned}
$$

From the fact that $F_{\lambda_{n}}(1 / n) \rightarrow+\infty$ as $n \rightarrow+\infty$, we obtain $\Psi_{1} \rightarrow-\infty$, that is, $\Psi_{1}$ is unbounded. This is a contradiction. 
Step 4. We show that $\Phi$ has a mountain pass geometry for $\lambda \leq \lambda_{0}$.

Fix $\lambda \in\left(0, \lambda_{0}\right]$. Then

$$
\begin{aligned}
F_{\lambda}(0) & =\int_{1}^{0} f_{\lambda}(s) d s=-\int_{0}^{1} f_{\lambda}(s) d s \\
& =-\int_{0}^{\lambda} f_{\lambda}(s) d s-\int_{\lambda}^{1} f_{\lambda}(s) d s \\
& =\frac{1}{\lambda^{\alpha-1}}-\int_{\lambda}^{1} f_{\lambda}(s) d s
\end{aligned}
$$

which implies that

$$
F_{\lambda}(0)>-\int_{\lambda}^{1} f_{\lambda}(s) d s=\int_{1}^{\lambda} f_{\lambda}(s) d s=F_{\lambda}(\lambda) .
$$

Hence

$$
\begin{aligned}
\Phi_{\lambda}(0) & =-F_{\lambda}(0) \int_{0}^{T} b(t) d t<-F_{\lambda}(\lambda) \int_{0}^{T} b(t) d t \\
& =-\frac{\int_{0}^{T} b(t) d t}{\alpha-1}\left(\frac{1}{\lambda^{\alpha-1}}-1\right) .
\end{aligned}
$$

Consider $\lambda \in\left(0, \lambda_{0}\right]$ such that

$$
\frac{1}{\lambda^{\alpha-1}}>1+\frac{d(\alpha-1)}{\int_{0}^{T} b(t) d t}
$$

Thus it follows from (3.8) that $\Phi_{\lambda}(0)<-d$.

Also, using $\left(\mathrm{S}_{3}\right)$, we can choose $R>1$ large enough that

$$
-\left(M(p-1)+\int_{0}^{T} e(t) d t\right) R-\frac{\int_{0}^{T} b(t) d t}{\alpha-1}\left(1-\frac{1}{R^{\alpha-1}}\right)>d .
$$

Then,

$$
\begin{aligned}
\Phi_{\lambda}(R) & =\sum_{j=1}^{p-1} \int_{0}^{R} I_{j}(s) d s-F_{\lambda}(R) \int_{0}^{T} b(t) d t+R \int_{0}^{T} e(t) d t \\
& \leq M(p-1) R+\frac{1}{\alpha-1}\left(1-\frac{1}{R^{\alpha-1}}\right) \int_{0}^{T} b(t) d t+R \int_{0}^{T} e(t) d t \\
& =\left(M(p-1)+\int_{0}^{T} e(t) d t\right) R+\frac{\int_{0}^{T} b(t) d t}{\alpha-1}\left(1-\frac{1}{R^{\alpha-1}}\right)<-d .
\end{aligned}
$$

Since $\Omega$ is a neighbourhood of $R, 0 \notin \Omega$ and

$$
\max \left\{\Phi_{\lambda}(0), \Phi_{\lambda}(R)\right\}<\inf _{x \in \partial \Omega} \Phi_{\lambda}(u) .
$$


Steps 1 and 2 imply that $\Phi_{\lambda}$ has a critical point $u_{\lambda}$ such that

$$
\Phi_{\lambda}\left(u_{\lambda}\right)=\inf _{h \in \Gamma} \max _{s \in[0,1]} \Phi_{\lambda}(h(s)) \geq \inf _{x \in \partial \Omega} \Phi_{\lambda}(u),
$$

where

$$
\Gamma=\left\{h \in C\left([0,1], H_{T}^{1}\right): h(0)=0, h(1)=R\right\} .
$$

Since $\inf _{u \in \partial \Omega} \Phi_{\lambda}\left(u_{\lambda}\right) \geq-d$, it follows from Step 3 that $u_{\lambda}$ is a solution of problem (1.1)-(1.2). The proof of the main result is complete.

\section{An example}

Consider the impulsive singular problem

$$
\begin{cases}u^{\prime \prime}-\frac{b(t)}{u^{\alpha}}=e(t), & \text { a.e. } t \in(0, T), \\ \Delta u^{\prime}\left(t_{j}\right)=I_{j}\left(u\left(t_{j}\right)\right), & j=1,2, \ldots, p-1, \\ u(0)-u(T)=u^{\prime}(0)-u^{\prime}(T)=0, & \end{cases}
$$

where $\alpha>1, p \geq 2$ and $T>0$. Take $b \in \mathbb{C}^{1}([0, T],(0, \infty))$ such that $b^{\prime}(t) \geq 0$ for all $t \in$ $[0, T], I_{j}(t)=\sin t, t \in \mathbb{R}$ and $e \in L^{2}([0, T], \mathbb{R})$ such that $\int_{0}^{T} e(t) d t<-(p-1)$. Choose $m=-1$ and $M=1$. Then $\left(\mathrm{S}_{1}\right)-\left(\mathrm{S}_{4}\right)$ in Theorem 1.1 hold. Therefore, problem (4.1) has at least one periodic solution.

\section{Acknowledgement}

The authors would like to thank Dr Jifeng Chu for some useful suggestions on this paper.

\section{References}

[1] R. P. Agarwal, D. Franco and D. O'Regan, 'Singular boundary value problems for first and second order impulsive differential equations', Aequationes Math. 69 (2005), 83-96.

[2] R. P. Agarwal and D. O'Regan, 'Existence criteria for singular boundary value problems with sign changing nonlinearities', J. Differential Equations 183 (2002), 409-433.

[3] R. P. Agarwal, K. Perera and D. O'Regan, 'Multiple positive solutions of singular problems by variational methods', Proc. Amer. Math. Soc. 134 (2005), 817-824.

[4] B. Ahmad and J. J. Nieto, 'Existence and approximation of solutions for a class of nonlinear impulsive functional differential equations with anti-periodic boundary conditions', Nonlinear Anal. 69 (2008), 3291-3298.

[5] A. Boucherif and N. Daoudi-Merzagui, 'Periodic solutions of singular nonautonomous second order differential equations', Nonlinear Differ. Equ. Appl. 15 (2008), 147-158.

[6] L. Chen and J. Sun, 'Nonlinear boundary value problem for first-order impulsive functional differential equations', J. Math. Anal. Appl. 318 (2006), 726-741.

[7] L. Chen, C. C. Tisdell and R. Yuan, 'On the solvability of periodic boundary value problems with impulse', J. Math. Anal. Appl. 331 (2007), 233-244.

[8] M. Choisy, J. F. Guégan and P. Rohani, 'Dynamics of infectious deseases and pulse vaccination: teasing apart the embedded resonance effects', Physica D 223 (2006), 26-35. 
[9] J. Chu, N. Fan and P. J. Torres, 'Periodic solutions for second order singular damped differential equations', J. Math. Anal. Appl. 388 (2012), 665-675.

[10] J. Chu, X. Lin, D. Jiang, D. O'Regan and P. R. Agarwal, 'Multiplicity of positive solutions to second order differential equations', Bull. Aust. Math. Soc. 73 (2006), 175-182.

[11] J. Chu and J. J. Nieto, 'Impulsive periodic solution of first-order singular differential equations', Bull. Lond. Math. Soc. 40 (2008), 143-150.

[12] J. Chu and D. O'Regan, 'Multiplicity results for second order nonautonomous singular Dirichlet systems', Acta Appl. Math. 105 (2009), 323-338.

[13] J. Chu, P. J. Torres and M. Zhang, 'Periodic solutions of second order nonautonomous singular dynamical systems', J. Differential Equations 239 (2007), 196-212.

[14] J. Chu and Z. Zhang, 'Periodic solutions of singular differential equations with sign-changing potential', Bull. Aust. Math. Soc. 82 (2010), 437-445.

[15] N. Daoudi-Merzagui, 'Periodic solutions of nonautonomous second order differential equations with a singularity', Appl. Anal. 73 (1999), 449-462.

[16] S. Gao, L. Chen, J. J. Nieto and A. Torres, 'Analysis of a delayed epidemic model with pulse vaccination and saturation incidence', Vaccine 24 (2006), 6037-6045.

[17] R. K. George, A. K. Nandakumaran and A. Arapostathis, 'A note on controllability of impulsive systems', J. Math. Anal. Appl. 241 (2000), 276-283.

[18] V. Lakshmikantham, D. D. Bainov and P. S. Simeonov, Theory of Impulsive Differential Equations (World Scientific, Singapore, 1989).

[19] W. Li, Y. Chang and J. J. Nieto, 'Solvability of impulsive neutral evolution differential inclusions with state-dependent delay', Math. Comput. Modelling 49 (2009), 1920-1927.

[20] J. Li and J. J. Nieto, 'Existence of positive solutions for multipoint boundary value problem on the half-line with impulses', Bound. Value Probl. (2009), (Article ID 834158, 12 pages).

[21] S. Nenov, 'Impulsive controllability and optimization problems in population dynamics', Nonlinear Anal. 36 (1999), 881-890.

[22] J. J. Nieto and D. O'Regan, 'Variational approach to impulsive differential equations', Nonlinear Anal. Real World Appl. 10 (2009), 680-690.

[23] J. Mawhin and M. Willem, Critical Point Theory and Hamiltonian Systems (Springer, Berlin, 1989).

[24] D. Qian and X. Li, 'Periodic solutions for ordinary differential equations with sublinear impulsive effects', J. Math. Anal. Appl. 303 (2005), 288-303.

[25] I. Rachunková and M. Tvrdý, 'Existence results for impulsive second-order periodic problems', Nonlinear Anal. 59 (2004), 133-146.

[26] J. Sun, H. Chen, J. J. Nieto and M. Otero-Novoa, 'Multiplicity of solutions for perturbed secondorder Hamiltonian systems with impulsive effects', Nonlinear Anal. 72 (2010), 4575-4586.

[27] Y. Tian and W. Ge, 'Applications of variational methods to boundary-value problem for impulsive differential equations', Proc. Edinb. Math. Soc. (2) 51 (2008), 509-527.

\title{
JUNTAO SUN, School of Science, Shandong University of Technology, Zibo, 255049 Shandong, China e-mail: sunjuntao2008@163.com
}

\author{
DONAL O'REGAN, Department of Mathematics, \\ National University of Ireland, Galway, Ireland \\ e-mail: donal.oregan@nuigalway.ie
}

